\title{
Diophantine sets. Preliminaries ${ }^{\rrbracket}$
}

\author{
Karol Pąk \\ Institute of Informatics \\ University of Białystok \\ Poland
}

\begin{abstract}
Summary. In this article, we define Diophantine sets using the Mizar formalism. We focus on selected properties of multivariate polynomials, i.e., functions of several variables to show finally that the class of Diophantine sets is closed with respect to the operations of union and intersection.

This article is the next in a series [1], [5] aiming to formalize the proof of Matiyasevich's negative solution of Hilbert's tenth problem.
\end{abstract}

MSC: 11D45 03B35

Keywords: Hilbert's 10th problem; Pell's equation; multivariate polynomials

MML identifier: HILB10_2, version: 8.1.07 5.47.1318

\section{INTRODUCTION}

Multivariate polynomials are often interpreted in informal mathematical practice as a finite sum of terms with each term being a product of a nonzero coefficient $c \in \mathfrak{F} \backslash\{\mathbf{0}\}$ and a monomial $x_{1}^{e_{1}} \cdot x_{2}^{e_{2}} \cdot \ldots \cdot x_{n}^{e_{n}}$ determined by an exponent vector $\left\langle e_{1}, e_{2}, \ldots, e_{n}\right\rangle \in \mathbb{N}^{n}$ where $n$ is a given natural number.

Formal interpretation of multivariate polynomials developed in the Mizar Mathematical Library [4] can be considered as a generalization of the informal approach, where the natural number $n$ is replaced by an ordinal number $\lambda$. Additionally, to avoid problems that occur when multiplying an infinite number of nonzero factors, each exponent vector $e: \lambda \mapsto \mathbb{N}$ has only a finite number of nonzero coordinates. Such exponent vectors are called bags of $\lambda$ and the set of all

\footnotetext{
${ }^{1}$ This work has been financed by the resources of the Polish National Science Centre granted by decision no. DEC-2015/19/D/ST6/01473. 
bags for a given $\lambda$ is denoted by Bags $\lambda$. It is important to note that for a finite $\lambda$, each bag $b$ corresponds to a finite sequence (with 0-based numbering). Using bags, multivariate polynomials have been defined in [6] as functions that assign a coefficient (an element of $\mathfrak{F}$ ) to each bag of $\lambda$ and are zero almost everywhere. Moreover, the evaluation for a multivariate polynomial $p$ and vector $x: \lambda \mapsto \mathfrak{F}$ has been defined as

$$
\operatorname{eval}(p, x)=\sum_{b \in \text { Bags } \lambda} p(b) \cdot \prod_{i \in \lambda} x(i)^{b(i)} .
$$

Based on this approach we define Diophantine sets in Def. 6 as follows. Let us consider a natural number $n$ that plays the role of the dimension and a subset $D$ of all finite sequences of length $n$ numbered from 0 (see Def. 5). We call $D$ Diophantine if there exist a natural number $k$ and a $n+k$-variable polynomial $p$ such that each coefficient is an integer number and

$$
\forall_{x: n \mapsto \mathbb{N}} x \in D \Longleftrightarrow \exists_{y: k \mapsto \mathbb{N}} \operatorname{eval}\left(p, x^{\frown} y\right)=0 .
$$

The main aim of our article is to show that the union and intersection of two $n$-dimension Diophantine sets $D_{1}, D_{2}$ is also Diophantine. The informal proof of these facts as presented by Z. Adamowicz and P. Zbierski in [2] or C. Smorynski [7] is quite obvious. Suppose that $p_{i}$ is $n+k_{i}$-variable polynomial which determines $D_{i}$ for $i=1,2$. Then $p_{1}^{\prime} \cdot p_{2}^{\prime}$ determines $D_{1} \cap D_{2}$ and $\left(p_{1}^{\prime}\right)^{2}+\left(p_{2}^{\prime}\right)^{2}$ determines $D_{1} \cup D_{2}$ where $p_{i}^{\prime}$ is the $n+k_{1}+k_{2}$-variable polynomial obtained from $p_{i}$ by modifying the order of variables and adding insignificant variables. The property of $p_{1}^{\prime}, p_{2}^{\prime}$, used in [2] can be formally formulated as follows:

$$
\left.\left.\operatorname{eval}\left(p_{1}^{\prime}, x^{\frown} y_{1}\right\urcorner y_{2}\right)=\operatorname{eval}\left(p_{1}, x^{\frown} y_{1}\right) \wedge \operatorname{eval}\left(p_{2}^{\prime}, x^{\frown} y_{1}\right\urcorner y_{2}\right)=\operatorname{eval}\left(p_{2}, x^{\frown} y_{2}\right)
$$

for arbitrary $x: n \mapsto \mathbb{N}, y_{1}: k_{1} \mapsto \mathbb{N}, y_{2}: k_{2} \mapsto \mathbb{N}$. The existence of such polynomials have been showed in Th. 27, 28, The construction of these polynomials is useful for the further development of multivariate polynomials in the Mizar Mathematical Library. Therefore we define and provide basic properties of two transformations that

- add an additional variable to the polynomial, preserving its value, i.e.

$$
\forall_{x: n \mapsto \mathbb{N}, a \in \mathbb{N}} \operatorname{eval}\left(p \text { extended by } 0, x^{\frown}\langle a\rangle\right)=\operatorname{eval}(p, x),
$$

- permute the order of variables, preserving its value, i.e.

$$
\forall x: n \mapsto \mathbb{N} \operatorname{eval}(p \text { permuted by } \sigma, x)=\operatorname{eval}\left(p, x \cdot \sigma^{-1}\right) .
$$




\section{Preliminaries}

From now on $i, j, k, n, m$ denote natural numbers and $b, b_{1}, b_{2}$ denote bags of $n$.

Let $X$ be a non empty set and $n$ be a natural number. Note that there exists a finite 0 -sequence of $X$ which is $n$-element and there exists a finite 0 -sequence which is $n$-element and real-valued.

Let $n, m$ be natural numbers, $p$ be an $n$-element finite 0 -sequence, and $q$ be an $m$-element finite 0 -sequence. One can check that $p^{\frown} q$ is $(n+m)$-element.

Let $p$ be a real-valued finite 0 -sequence and $q$ be a real-valued finite 0 sequence. Let us observe that $p^{\frown} q$ is real-valued.

Let $n$ be a natural number and $p$ be an $n$-element, real-valued finite 0 sequence. The functor ${ }^{\circledR} p$ yielding a function from $n$ into $\mathbb{R}_{\mathrm{F}}$ is defined by the term

(Def. 1) $p$.

Let $X$ be a non empty set and $p$ be a function from $n$ into $X$. The functor ${ }^{\circledR} p$ yielding an $n$-element finite 0 -sequence of $X$ is defined by the term

(Def. 2) $p$.

Let $X$ be a set, $p$ be a permutation of $X$, and $M$ be a many sorted set indexed by $X$. Observe that $M \cdot p$ is total.

Let $F$ be a finite-support function and $f$ be a one-to-one function. Let us observe that $F \cdot f$ is finite-support.

Now we state the propositions:

(1) Let us consider finite 0 -sequences $F, G$. Suppose $F^{\frown} G$ is one-to-one. Then $\operatorname{rng} F$ misses $\operatorname{rng} G$.

(2) Let us consider a set $X$, an $X$-defined function $f$, and a permutation $\sigma$ of $X$. Then $\overline{\overline{\text { support } f \cdot \sigma}}=\overline{\overline{\text { support } f}}$.

Proof: Set $P=\sigma^{-1} \cdot P^{\circ}(\operatorname{support} f) \subseteq \operatorname{support} f \cdot \sigma$. support $f \cdot \sigma \subseteq$ $P^{\circ}$ (support $f$ ).

Let $X$ be a set. Observe that $0_{X}\left(\mathbb{R}_{\mathrm{F}}\right)$ is natural-valued and $1_{-}\left(X, \mathbb{R}_{\mathrm{F}}\right)$ is natural-valued.

Let $x$ be an element of $X$. Note that $1 \_1\left(x, \mathbb{R}_{\mathrm{F}}\right)$ is natural-valued and there exists a series of $X, \mathbb{R}_{\mathrm{F}}$ which is $\mathbb{Z}$-valued.

Let $O$ be an ordinal number. Let us note that there exists a polynomial of $O, \mathbb{R}_{\mathrm{F}}$ which is $\mathbb{Z}$-valued.

Let $X$ be a set and $p$ be a $\mathbb{Z}$-valued series of $X, \mathbb{R}_{\mathrm{F}}$. One can check that $-p$ is $\mathbb{Z}$-valued.

Let $q$ be a $\mathbb{Z}$-valued series of $X, \mathbb{R}_{\mathrm{F}}$. Observe that $p+q$ is $\mathbb{Z}$-valued and $p-q$ is $\mathbb{Z}$-valued. 
Let $X$ be an ordinal number and $p, q$ be $\mathbb{Z}$-valued series of $X, \mathbb{R}_{\mathrm{F}}$. One can verify that $p * q$ is $\mathbb{Z}$-valued.

Let $X$ be a set. Let us note that there exists a function from $X$ into $\mathbb{R}_{F}$ which is natural-valued.

Let $O$ be an ordinal number. One can check that there exists a function from $O$ into $\mathbb{R}_{\mathrm{F}}$ which is $\mathbb{Z}$-valued.

Let $b$ be a bag of $O$ and $x$ be a $\mathbb{Z}$-valued function from $O$ into $\mathbb{R}_{\mathrm{F}}$. Note that $\operatorname{eval}(b, x)$ is integer.

Let $p$ be a $\mathbb{Z}$-valued polynomial of $O, \mathbb{R}_{\mathrm{F}}$. One can check that eval $(p, x)$ is integer.

\section{Polynomial Extended By 0}

Now we state the propositions:

(3) Let us consider a many sorted set $b$ indexed by $n$. If $k \leqslant n$, then $\langle b(0), \ldots, b(k)\rangle=b\lceil k$.

Proof: For every object $x$ such that $x \in k$ holds $\langle b(0), \ldots, b(k)\rangle(x)=$ $(b \nmid k)(x)$.

(4) Let us consider a bag $b$ of $n+1$. Then $b=\langle b(0), \ldots, b(n)\rangle$ extended by $b(n)$. Proof: Set $C=\langle b(0), \ldots, b(n)\rangle$. Set $B=C$ extended by $b(n) . C=b \nmid n$. For every object $x$ such that $x \in n+1$ holds $B(x)=b(x)$ by [8, (2)].

(5) $\langle b$ extended by $k(0), \ldots, b$ extended by $k(n)\rangle=b$. The theorem is a consequence of (3).

Let us consider $n$. Let $L$ be a non empty zero structure and $p$ be a series of $n, L$. The $p$ extended by 0 yielding a series of $n+1, L$ is defined by

(Def. 3) for every bag $b$ of $n+1$, if $b(n) \neq 0$, then it $(b)=0_{L}$ and if $b(n)=0$, then $i t(b)=p(\langle b(0), \ldots, b(n)\rangle)$.

Now we state the propositions:

(6) Let us consider a non empty zero structure $L$, and a series $p$ of $n, L$. Then (the $p$ extended by 0$)(b$ extended by 0$)=p(b)$. The theorem is a consequence of (5).

(7) Let us consider a non empty zero structure $L$, a series $p$ of $n, L$, and a bag $b$ of $n+1$. Suppose $b \in \operatorname{Support}($ the $p$ extended by 0 ). Then $b(n)=0$.

(8) Let us consider a non empty zero structure $L$, and a series $p$ of $n, L$. Then $b$ extended by $0 \in \operatorname{Support}($ the $p$ extended by 0 ) if and only if $b \in$ Support $p$. The theorem is a consequence of (5).

(9) Let us consider a non empty zero structure $L$, a series $p$ of $n, L$, and a bag $b$ of $n+1$. Suppose $b(n)=0$. Then $b \in \operatorname{Support}($ the $p$ extended by 0 ) 
if and only if $\langle b(0), \ldots, b(n)\rangle \in \operatorname{Support} p$. The theorem is a consequence of (4) and (8).

Let us consider $n$. Let $L$ be a non empty zero structure and $p$ be a polynomial of $n, L$. Let us observe that the $p$ extended by 0 is finite-Support.

Now we state the propositions:

(10) Let us consider a non empty zero structure $L$, and a series $p$ of $n, L$. Then $\left\{0_{L}\right\} \cup \operatorname{rng} p=\operatorname{rng}($ the $p$ extended by 0$)$. The theorem is a consequence of (6).

(11) $\operatorname{support} b=\operatorname{support}(b$ extended by 0$)$.

Proof: Set $E=b$ extended by 0 . support $b \subseteq \operatorname{support} E$.

(12) $\operatorname{SgmX}\left(\subseteq_{n}, \operatorname{support} b\right)=\operatorname{SgmX}\left(\subseteq_{n+1}, \operatorname{support}(b\right.$ extended by 0$\left.)\right)$. The theorem is a consequence of (11).

(13) Let us consider a well unital, non trivial double loop structure $L$, a function $x$ from $n$ into $L$, and a function $y$ from $n+1$ into $L$. Suppose $y\lceil n=x$. Then eval $(b, x)=\operatorname{eval}(b$ extended by $0, y)$.

Proof: Set $S=\operatorname{SgmX}\left(\subseteq_{n}\right.$, support $\left.b\right)$. Set $B=b$ extended by 0 . Set $S_{1}=$ $\operatorname{SgmX}\left(\subseteq_{n+1}\right.$, support $\left.B\right)$. Consider $P$ being a finite sequence of elements of $L$ such that len $P=$ len $S$ and $\operatorname{eval}(b, x)=\prod P$ and for every element $i$ of $\mathbb{N}$ such that $1 \leqslant i \leqslant \operatorname{len} P$ holds $P_{/ i}=\operatorname{power}_{L}\left(x \cdot S_{/ i}, b \cdot S_{/ i}\right)$. Consider $P_{1}$ being a finite sequence of elements of $L$ such that len $P_{1}=\operatorname{len} S_{1}$ and $\operatorname{eval}(B, y)=\prod P_{1}$ and for every element $i$ of $\mathbb{N}$ such that $1 \leqslant i \leqslant \operatorname{len} P_{1}$ holds $P_{1 / i}=\operatorname{power}_{L}\left(y \cdot S_{1 / i}, B \cdot S_{1 / i}\right) . S=S_{1} \cdot b=\langle B(0), \ldots, B(n)\rangle$. For every natural number $i$ such that $1 \leqslant i \leqslant \operatorname{len} P$ holds $P(i)=P_{1}(i)$.

(14) $b_{1}<b_{2}$ if and only if $b_{1}$ extended by $k<b_{2}$ extended by $k$.

Proof: Set $B_{1}=b_{1}$ extended by $k$. Set $B_{2}=b_{2}$ extended by $k$. If $b_{1}<b_{2}$, then $b_{1}$ extended by $k<b_{2}$ extended by $k$. Consider $o$ being an ordinal number such that $B_{1}(o)<B_{2}(o)$ and for every ordinal number $l$ such that $l \in o$ holds $B_{1}(l)=B_{2}(l)$. For every ordinal number $l$ such that $l \in o$ holds $b_{1}(l)=b_{2}(l)$.

(15) Let us consider a non empty set $X$, a finite subset $A$ of $X$, and an order $R$ in $X$. Suppose $R$ linearly orders $A$. Suppose $1 \leqslant i \leqslant k \leqslant \overline{\bar{A}}$. Then $\left(\operatorname{SgmX}(R, \operatorname{rng}(\operatorname{SgmX}(R, A)\lceil k)))_{i i}=(\operatorname{SgmX}(R, A))_{/ i}\right.$.

Proof: Set $S_{1}=\operatorname{SgmX}(R, A)$. Define $\mathcal{P}$ [natural number] $\equiv$ for every $i$ such that $1 \leqslant i \leqslant \$_{1} \leqslant \overline{\bar{A}} \operatorname{holds}\left(\operatorname{SgmX}\left(R, \operatorname{rng}\left(S_{1} \uparrow \$_{1}\right)\right)\right)_{/ i}=S_{1 / i}$. For every $k$ such that $\mathcal{P}[k]$ holds $\mathcal{P}[k+1]$ by $[3$, (83)]. For every $k, \mathcal{P}[k]$.

(16) Let us consider an ordinal number $O$, and a finite subset $A$ of $\operatorname{Bags} O$. Suppose $n, m \in \operatorname{dom} \operatorname{SgmX}(\operatorname{Bag} O r d e r O, A)$ and $n<m$.

Then $(\operatorname{SgmX}(\operatorname{BagOrder} O, A))_{/ n}<(\operatorname{SgmX}(\operatorname{BagOrder} O, A))_{/ m}$. 
(17) Let us consider a right zeroed, add-associative, right complementable, well unital, distributive, non trivial double loop structure $L$, and a polynomial $p$ of $n, L$. Then

(i) $\operatorname{len} \operatorname{SgmX}(\operatorname{BagOrder} n, \operatorname{Support} p)=$

len $\operatorname{SgmX}(\operatorname{BagOrder}(n+1)$, Support(the $p$ extended by 0$))$, and

(ii) for every natural number $i$ such that

$1 \leqslant i \leqslant \operatorname{len} \operatorname{SgmX}($ BagOrder $n$, Support $p)$ holds

$(\operatorname{SgmX}(\operatorname{BagOrder}(n+1), \operatorname{Support}(\text { the } p \text { extended by } 0)))_{/ i}=$

$(\operatorname{SgmX}(\operatorname{BagOrder} n, \operatorname{Support} p))_{/ i}$ extended by 0 .

Proof: Set $B=\operatorname{BagOrder} n$. Set $B_{1}=\operatorname{BagOrder}(n+1)$. Set $P=$ the $p$ extended by 0 . Define $\mathcal{F}($ bag of $n)=\$_{1}$ extended by 0 . Consider $f$ being a function from Bags $n$ into $\operatorname{Bags}(n+1)$ such that for every element $x$ of Bags $n, f(x)=\mathcal{F}(x)$. Set $F=f\left\lceil\right.$ Support $p$. Set $S_{1}=$ $\operatorname{SgmX}(B, \operatorname{Support} p)$. Set $S_{2}=\operatorname{SgmX}\left(B_{1}, \operatorname{Support} P\right) \operatorname{rng} F \subseteq \operatorname{Support} P$. Support $P \subseteq \operatorname{rng} F . F$ is one-to-one. Define $\mathcal{P}$ [natural number] $\equiv$ if $1 \leqslant$ $\$_{1} \leqslant$ len $S_{1}$, then for every $i$ such that $1 \leqslant i \leqslant \$_{1}$ holds $S_{2 / i}=S_{1 / i}$ extended by 0 . For every natural number $k$ such that $\mathcal{P}[k]$ holds $\mathcal{P}[k+1]$. For every $k, \mathcal{P}[k]$.

(18) Let us consider a right zeroed, add-associative, right complementable, well unital, distributive, non trivial double loop structure $L$, a polynomial $p$ of $n, L$, a function $x$ from $n$ into $L$, and a function $y$ from $n+1$ into $L$. Suppose $y\lceil n=x$. Then eval $(p, x)=\operatorname{eval}($ the $p$ extended by $0, y)$.

Proof: Set $n_{1}=n+1$. Set $S=\operatorname{SgmX}(\operatorname{BagOrder} n, \operatorname{Support} p)$. Set $P=$ the $p$ extended by 0 . Set $S_{1}=\operatorname{SgmX}\left(\operatorname{BagOrder} n_{1}\right.$, Support $\left.P\right)$. Consider $T$ being a finite sequence of elements of $L$ such that len $T=\operatorname{len} S$ and $\operatorname{eval}(p, x)=\sum T$ and for every element $i$ of $\mathbb{N}$ such that $1 \leqslant i \leqslant \operatorname{len} T$ holds $T_{/ i}=p \cdot S_{/ i} \cdot \operatorname{eval}\left(S_{/ i}, x\right)$. Consider $T_{1}$ being a finite sequence of elements of $L$ such that len $T_{1}=$ len $S_{1}$ and eval $(P, y)=\sum T_{1}$ and for every element $i$ of $\mathbb{N}$ such that $1 \leqslant i \leqslant \operatorname{len} T_{1}$ holds $T_{1 / i}=P \cdot S_{1 / i} \cdot \operatorname{eval}\left(S_{1 / i}, y\right)$. len $S=$ len $S_{1}$ and for every natural number $i$ such that $1 \leqslant i \leqslant \operatorname{len} S$ holds $S_{1 / i}=S_{/ i}$ extended by 0 . For every natural number $i$ such that $1 \leqslant i \leqslant \operatorname{len} S$ holds $T(i)=T_{1}(i)$.

\section{Polynomial Permuted by Permutation}

Now we state the propositions:

(19) Let us consider an ordinal number $O$, a well unital, commutative, associative, non trivial double loop structure $L$, a function $x$ from $O$ into $L$, a bag $b$ of $O$, and a one-to-one finite sequence $S$ of elements of $O$. Suppose 
$\operatorname{rng} S=\operatorname{support} b$. Then there exists a finite sequence $y$ of elements of $L$ such that

(i) len $y=\overline{\overline{\text { support } b}}$, and

(ii) $\operatorname{eval}(b, x)=\prod y$, and

(iii) for every $i$ such that $1 \leqslant i \leqslant \operatorname{len} y$ holds $y_{/ i}=\operatorname{power}_{L}\left(x \cdot S_{/ i}, b \cdot S_{/ i}\right)$.

(20) Let us consider an ordinal number $O$, a well unital, commutative, associative, non trivial double loop structure $L$, a function $x$ from $O$ into $L$, a bag $b$ of $O$, and a permutation $\sigma$ of $O$. Then $\operatorname{eval}(b, x)=\operatorname{eval}(b \cdot \sigma, x \cdot \sigma)$. Proof: Set $S_{1}=\operatorname{SgmX}\left(\subseteq_{n}\right.$, support $\left.b\right)$. Consider $y$ being a finite sequence of elements of $L$ such that len $y=\operatorname{len} S_{1}$ and eval $(b, x)=\prod y$ and for every element $i$ of $\mathbb{N}$ such that $1 \leqslant i \leqslant \operatorname{len} y$ holds $y_{/ i}=\operatorname{power}_{L}\left(x \cdot S_{1 / i}, b \cdot S_{1 / i}\right)$. Set $P=\sigma^{-1}$. $\operatorname{rng} P \cdot S_{1} \subseteq \operatorname{support} b \cdot \sigma$. support $b \cdot \sigma \subseteq \operatorname{rng} P \cdot S_{1}$. Reconsider $S=P \cdot S_{1}$ as a one-to-one finite sequence of elements of $n$. Consider $Y$ being a finite sequence of elements of $L$ such that len $Y=\overline{\overline{\text { support } b \cdot \sigma}}$ and eval $(b \cdot \sigma, x \cdot \sigma)=\prod Y$ and for every natural number $i$ such that $1 \leqslant i \leqslant \operatorname{len} Y$ holds $Y_{/ i}=\operatorname{power}_{L}\left(x \cdot \sigma \cdot S_{/ i}, b \cdot \sigma \cdot S_{/ i}\right)$. len $Y=\operatorname{len} y$. For every natural number $i$ such that $1 \leqslant i \leqslant \operatorname{len} Y$ holds $Y(i)=y(i)$.

Let $O$ be an ordinal number, $L$ be a non empty zero structure, $s$ be a series of $O, L$, and $\sigma$ be a permutation of $O$. The $s$ permuted by $\sigma$ yielding a series of $O, L$ is defined by

(Def. 4) for every bag $b$ of $O, i t(b)=s(b \cdot \sigma)$.

Let us consider an ordinal number $O$, a non empty zero structure $L$, a permutation $\sigma$ of $O$, a series $s$ of $O, L$, and a bag $b$ of $O$. Now we state the propositions:

(21) $b \in \operatorname{Support}($ the $s$ permuted by $\sigma$ ) if and only if $b \cdot \sigma \in \operatorname{Support} s$.

(22) $b \cdot \sigma^{-1} \in \operatorname{Support}($ the $s$ permuted by $\sigma$ ) if and only if $b \in$ Support $s$.

(23) Let us consider an ordinal number $O$, a non empty zero structure $L$, a permutation $\sigma$ of $O$, and a series $s$ of $O, L$. Then $\overline{\overline{\text { Support } s}}=\overline{\overline{\text { Support } \alpha}}$, where $\alpha$ is the $s$ permuted by $\sigma$.

Proof: Set $P=$ the $s$ permuted by $\sigma$. Define $\mathcal{R}[$ bag of $O$, bag of $O] \equiv$ $\$_{2}=\$_{1} \cdot \sigma$. For every element $x$ of $\operatorname{Bags} O$, there exists an element $y$ of $\operatorname{Bags} O$ such that $\mathcal{R}[x, y]$. Consider $f$ being a function from Bags $O$ into $\operatorname{Bags} O$ such that for every element $x$ of $\operatorname{Bags} O, \mathcal{R}[x, f(x)] . f$ is one-to-one. $f^{\circ}($ Support $P) \subseteq$ Support $s$. Support $s \subseteq f^{\circ}($ Support $P)$.

(24) Let us consider an ordinal number $O$, an Abelian, right zeroed, addassociative, right complementable, well unital, distributive, non trivial double loop structure $L$, a polynomial $p$ of $O, L$, a function $x$ from $O$ into $L$, and a one-to-one finite sequence $S$ of elements of $\operatorname{Bags} O$. Suppose 
$\operatorname{rng} S=\operatorname{Support} p$. Then there exists a finite sequence $y$ of elements of $L$ such that

(i) len $y=\overline{\overline{\text { Support } p}}$, and

(ii) $\operatorname{eval}(p, x)=\sum y$, and

(iii) for every natural number $i$ such that $1 \leqslant i \leqslant \operatorname{len} y$ holds $y_{/ i}=$ $p \cdot S_{/ i} \cdot \operatorname{eval}\left(S_{/ i}, x\right)$.

Let $O$ be an ordinal number, $L$ be a non empty zero structure, $\sigma$ be a permutation of $O$, and $p$ be a polynomial of $O, L$. One can check that the $p$ permuted by $\sigma$ is finite-Support.

(25) Let us consider an ordinal number $O$, an Abelian, right zeroed, addassociative, right complementable, well unital, distributive, commutative, associative, non trivial double loop structure $L$, a polynomial $p$ of $O, L$, a function $x$ from $O$ into $L$, and a permutation $\sigma$ of $O$. Then $\operatorname{eval}(p, x)=\operatorname{eval}\left(\right.$ the $p$ permuted by $\left.\sigma, x \cdot\left(\sigma^{-1}\right)\right)$.

Proof: Set $S_{2}=\operatorname{SgmX}(\operatorname{BagOrder} O, \operatorname{Support} p)$. Consider $y$ being a finite sequence of elements of $L$ such that len $y=\operatorname{len} S_{2}$ and $\operatorname{eval}(p, x)=\sum y$ and for every element $i$ of $\mathbb{N}$ such that $1 \leqslant i \leqslant \operatorname{len} y$ holds $y_{/ i}=p \cdot S_{2 / i}$. $\operatorname{eval}\left(S_{2 / i}, x\right)$. Set $P=$ the $p$ permuted by $\sigma$. Define $\mathcal{R}[$ bag of $O$, bag of $O] \equiv \$_{2}=\$_{1} \cdot \sigma^{-1}$. For every element $x$ of $\operatorname{Bags} O$, there exists an element $y$ of $\operatorname{Bags} O$ such that $\mathcal{R}[x, y]$. Consider $f$ being a function from $\operatorname{Bags} O$ into Bags $O$ such that for every element $x$ of $\operatorname{Bags} O, \mathcal{R}[x, f(x)] . f$ is oneto-one. Reconsider $f_{1}=f \cdot S_{2}$ as a one-to-one finite sequence of elements of Bags $O . \operatorname{rng} f_{1} \subseteq \operatorname{Support} P$. Support $P \subseteq \operatorname{rng} f_{1}$. Consider $z$ being a finite sequence of elements of $L$ such that len $z=\overline{\overline{\text { Support } P}}$ and eval $(P, x$. $\left.\sigma^{-1}\right)=\sum z$ and for every natural number $i$ such that $1 \leqslant i \leqslant \operatorname{len} z$ holds $z_{/ i}=P \cdot f_{1 / i} \cdot \operatorname{eval}\left(f_{1 / i}, x \cdot \sigma^{-1}\right)$. len $y=\operatorname{len} z$. For every natural number $i$ such that $1 \leqslant i \leqslant \operatorname{len} y$ holds $y(i)=z(i)$.

(26) Let us consider an ordinal number $O$, a non empty zero structure $L$, a series $s$ of $O, L$, and a permutation $\sigma$ of $O$. Then $\operatorname{rng}($ the $s$ permuted by $\sigma)=\operatorname{rng} s$.

\section{Main Lemmas}

Now we state the propositions:

(27) Let us consider a right zeroed, add-associative, right complementable, well unital, distributive, non trivial double loop structure $L$, and a polynomial $p$ of $n, L$. Then there exists a polynomial $q$ of $n+m, L$ such that

(i) $\operatorname{rng} q \subseteq \operatorname{rng} p \cup\left\{0_{L}\right\}$, and 
(ii) for every function $x$ from $n$ into $L$ and for every function $y$ from $n+m$ into $L$ such that $y\lceil n=x \operatorname{holds} \operatorname{eval}(p, x)=\operatorname{eval}(q, y)$.

Proof: Define $\mathcal{P}$ [natural number] $\equiv$ there exists a polynomial $q$ of $n+\$_{1}, L$ such that $\operatorname{rng} q \subseteq \operatorname{rng} p \cup\left\{0_{L}\right\}$ and for every function $x$ from $n$ into $L$ and for every function $y$ from $n+\$_{1}$ into $L$ such that $y\lceil n=x$ holds $\operatorname{eval}(p, x)=\operatorname{eval}(q, y) \cdot \mathcal{P}[0]$. If $\mathcal{P}[k]$, then $\mathcal{P}[k+1] . \mathcal{P}[k]$.

(28) Let us consider an Abelian, right zeroed, add-associative, right complementable, well unital, distributive, commutative, associative, non trivial double loop structure $L$, and a polynomial $p$ of $n+m, L$. Then there exists a polynomial $q$ of $n+k+m, L$ such that

(i) $\operatorname{rng} q \subseteq \operatorname{rng} p \cup\left\{0_{L}\right\}$, and

(ii) for every function $X_{1}$ from $n+m$ into $L$ and for every function $X_{2}$ from $n+k+m$ into $L$ such that $X_{1}\left\lceil n=X_{2}\left\lceil n\right.\right.$ and $\left({ }^{@} X_{1}\right)_{\downarrow n}=$ $\left({ }^{@} X_{2}\right)_{\lfloor n+k} \operatorname{holds} \operatorname{eval}\left(p, X_{1}\right)=\operatorname{eval}\left(q, X_{2}\right)$.

Proof: Consider $P$ being a polynomial of $n+m+k, L$ such that $\operatorname{rng} P \subseteq$ $\operatorname{rng} p \cup\left\{0_{L}\right\}$ and for every function $x$ from $n+m$ into $L$ and for every function $y$ from $n+m+k$ into $L$ such that $y\lceil(n+m)=x \operatorname{holds} \operatorname{eval}(p, x)=$ eval $(P, y)$. Reconsider $P_{1}=P$ as a polynomial of $n+k+m, L$. Set $I=$ $\operatorname{id}_{n+k+m}$. Set $n_{1}=n+m$. Set $I_{2}=I\left\lceil n_{1} \cdot \operatorname{rng} I_{2} \operatorname{misses} \operatorname{rng} I_{\left\lfloor n_{1}\right.} \cdot \operatorname{rng}\left(I_{2}\lceil n)\right.\right.$ misses $\operatorname{rng} I_{2\lfloor n}$. Reconsider $I_{1}=\left(\left(I_{2}\lceil n)^{\frown} I_{\left\lfloor n_{1}\right.}\right)^{\frown} I_{2 \downarrow n}\right.$ as a function from $n+k+m$ into $n+k+m$. Reconsider $R={ }^{@} X_{1} \frown\left({ }^{@} X_{2} \uparrow(n+k)\right)_{l n}$ as a function from $n+m+k$ into $L$. Reconsider $r=R$ as a function from $n+k+m$ into $L$. eval $\left(P_{1}, r\right)=\operatorname{eval}\left(T, r \cdot I_{1}\right)$. For every $k$ such that $k \in \operatorname{dom}{ }^{@} X_{2}$ holds $\left({ }^{@} r \cdot I_{1}\right)(k)=\left({ }^{@} X_{2}\right)(k)$.

\section{Diophantine Sets}

From now on $x, s$ denote objects.

Let $D$ be a non empty set and $n$ be a natural number. The $n$-xtuples of $D$ yielding a subset of $D^{\omega}$ is defined by

(Def. 5) $\quad x \in$ it iff $x$ is an $n$-element finite 0 -sequence of $D$.

Observe that the $n$-xtuples of $D$ is non empty and every element of the $n$ xtuples of $D$ is $n$-element and $D$-valued.

Let $A$ be a subset of the $n$-xtuples of $\mathbb{N}$. We say that $A$ is diophantine if and only if

(Def. 6) there exists a natural number $m$ and there exists a $\mathbb{Z}$-valued polynomial $p$ of $n+m, \mathbb{R}_{\mathrm{F}}$ such that for every $s, s \in A$ iff there exists an $n$-element 
finite 0 -sequence $x$ of $\mathbb{N}$ and there exists an $m$-element finite 0 -sequence $y$ of $\mathbb{N}$ such that $s=x$ and $\operatorname{eval}\left(p,{ }^{\circledR}\left(x^{\frown} y\right)\right)=0$.

One can verify that every subset of the $n$-xtuples of $\mathbb{N}$ which is empty is also diophantine and $\Omega_{\text {the } n \text {-xtuples of } \mathbb{N}}$ is diophantine.

Let $n$ be a zero natural number. One can verify that every subset of the $n$ xtuples of $\mathbb{N}$ is diophantine.

Let $n$ be a natural number. Let us observe that there exists a subset of the $n$ xtuples of $\mathbb{N}$ which is non empty and diophantine and there exists a subset of the $n$-xtuples of $\mathbb{N}$ which is empty and diophantine.

Let $A, B$ be diophantine subsets of the $n$-xtuples of $\mathbb{N}$. One can check that $A \cap B$ is diophantine as a subset of the $n$-xtuples of $\mathbb{N}$ and $A \cup B$ is diophantine as a subset of the $n$-xtuples of $\mathbb{N}$.

\section{REFERENCES}

[1] Marcin Acewicz and Karol Pak. Pell's equation. Formalized Mathematics, 25(3):197-204, 2017. doi $10.1515 /$ forma-2017-0019

[2] Zofia Adamowicz and Paweł Zbierski. Logic of Mathematics: A Modern Course of Classical Logic. Pure and Applied Mathematics: A Wiley Series of Texts, Monographs and Tracts. Wiley-Interscience, 1997.

[3] Czesław Byliński. Some properties of restrictions of finite sequences. Formalized Mathematics, 5(2):241-245, 1996.

[4] Adam Grabowski, Artur Korniłowicz, and Adam Naumowicz. Four decades of Mizar. Journal of Automated Reasoning, 55(3):191-198, 2015. doi:10.1007/s10817-015-9345-1

[5] Karol Pąk. The Mativasevich theorem. Preliminaries. Formalized Mathematics, 25(4): 315-322, 2017. doi $10.1515 /$ forma-2017-0029

[6] Piotr Rudnicki and Andrzej Trybulec. Multivariate polynomials with arbitrary number of variables Formalized Mathematics, 9(1):95-110, 2001.

[7] Craig Smorynski. Logical Number Theory I, An Introduction. Universitext. Springer-Verlag Berlin Heidelberg, 1991. ISBN 978-3-642-75462-3.

[8] Tetsuya Tsunetou, Grzegorz Bancerek, and Yatsuka Nakamura. Zero-based finite sequences Formalized Mathematics, 9(4):825-829, 2001. 